\title{
VR Teleoperation to support a GPS-free Positioning System in a Marine Environment
}

\author{
M. Lager, E.A. Topp \& J.Malec \\ Lund University, Lund, Sweden
}

\begin{abstract}
Small autonomous surface vehicles (ASV) will need both teleoperation support and redundant positioning technology to comply with expected future regulations. When at sea, they are limited by a satellite communication link with low throughput. We have designed and implemented a graphical user interface (GUI) for teleoperation using a communication link with low throughput, and one positioning system, independent of the Global Positioning System (GPS), supported by the teleoperation tool. We conducted a user study (N=16), using real-world data from a field trial, to validate our approach, and to compare two variants of the graphical user interface (GUI). The users experienced that the tool gives a good overview, and despite the connection with the low throughput, they managed through the GUI to significantly improve the positioning accuracy.
\end{abstract}

\section{INTRODUCTION}

Autonomy and artificial intelligence are disrupting many sectors, including the marine industry. Many companies and academia are researching to evolve the field. Some companies have even started testing autonomy in real commercial routes (with safety drivers on board to meet current regulations). In late 2018 a ferry, developed by Finferries and Rolls Royce, went between two cities in Finland, first navigating autonomously and then remotely operated when returning [1]. In Norway, also in 2018, Kongsberg started testing autonomy on an autonomous ferry with passengers and cars on board, mainly to reduce the workload and to increase the safety [2]. To convince authorities to change regulations to permit using ships without a crew on board, it is of utmost importance to guarantee safety. A human onboard a ship is very flexible, and will in many situations discover if the ship is behaving strangely or if an unexpected event arises. When removing the crew, the vessel will need to incorporate this extra safety feature into the system instead.

When it comes to safe navigation, to have a correct position is vital. Nowadays, crew members rely heavily on the Global Positioning System (GPS) for this. A loss of the GPS signal, or a jammed or spoofed GPS, can for a crew-less ship result in hazardous situations. The global quality assurance and risk management company DNV GL believes unmanned ships may need alternative positioning methods to convince authorities that their safety is satisfactory [3]. Furthermore, they believe autonomous ships will not be fully autonomous for many years, but instead rely on autonomy and remote control in combination. Rolls Royce also believes this, as they see the teleoperation of ships as a key technology in the transferring process towards autonomous ships [4]. Moreover, they claim that the teleoperation of an autonomous vessel will increase reliability and performance. The communication link for the teleoperation system is vulnerable to downtime, 
though, and during this time, the system must solve the actions autonomously.

The work described by this paper has focused on how to use remote operation to improve positioning accuracy for small affordable vessels. Unmanned ground vehicles (UGV) have, for many years, been teleoperated to master harsh environments during, e.g., military or search and rescue (SAR) missions [5][7]. Small autonomous vessels at sea are also essential, and Murphy believes they will play an important role during future SAR operations [8]. The challenges with remote control and positioning are similar for small and large ships. However, the communication link's throughput sets a limitation on smaller, more affordable vessels, as they can not have a large satellite antenna due to the size, weight, and cost constraints. This limitation makes the streaming of video and transmission of high-resolution images infeasible. For the positioning problem, we have, for the same reason, confined ourselves only to use affordable navigation sensors.

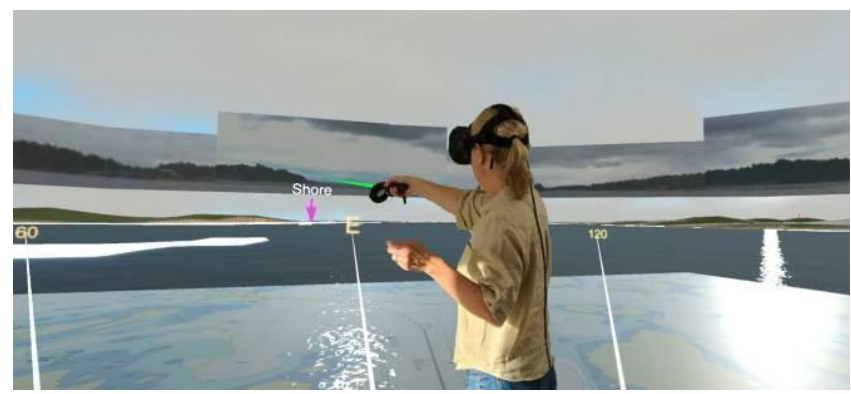

Figure 1. A participant of the user study taking a bearing by pointing towards an augmented landmark.

The positioning system is built upon our previous implementation with terrain-aided navigation (TAN), presented in [9]. This paper estimated the position from a real-world field trial by comparing the bottom depth and magnetic intensity with available maps. To enhance the position accuracy even further, we manually measured bearings to landmarks from the recorded $360^{\circ}$ image, making it possible for the positioning tool to adjust the position estimation accordingly. This is not possible to do manually on an unmanned ship. In this new work, a user instead measures these bearings from a teleoperation system in virtual reality (VR), see Figure 1.

The teleoperation system also builds on our previous work, presented in [10], [11]. This work focused on developing a teleoperation tool with a low-cognitive load that could provide a good situational awareness (SA), leading to better safety for the vessel. In the work described in the latter paper, we developed a specific GUI to compare the performance when using VR, 3D visualization on a laptop, and 2D visualization on a laptop. In this earlier study, we observed that the longer available time for decisions at sea, measured in seconds or minutes, makes it ideal for teleoperation. This contrasts with the fast dynamics of the traffic situations for cars and airplanes, often measured in milliseconds, reported as challenging teleoperation areas due to the vulnerability from mainly long latency [12], [13]. Several research papers propose methods to compensate or predict the teleoperated vehicle's pose to mitigate the latency problem [14]-
[16]. We use this knowledge to predict our current position based on heading, speed, and the received estimated position from the remote vessel. We concluded in our previous study that 3D, and especially VR, gave the best performance. VR can strengthen the visualization, and thereby the total communication between the machine and the human [17]. It has also been shown that VR can enhance SA when driving a remote car [18], [19]. Because of our good results for VR in our previous work, we use only VR in this current work. Here we have re-built the GUI to evaluate how teleoperation can support navigation, and more specifically, the TAN application. We base the user evaluation on recordings from a field trial to make the user experience as realistic as possible.

One of our main objectives has been to provide the user with an immersive experience that provides good SA. To gain trust in the system's ability to navigate, it is essential that the user gets a good overview and instantly can determine whether the position is estimated correctly or not. When navigating onboard a manned vessel, the usual way of doing this is to try to match the real-world terrain with objects on the sea chart or radar and try to judge if the directions and ranges coincide. The mental rotations needed for this task are difficult for a human to perform [20], [21], and we believe it is even more challenging to do remotely, i.e., by comparing what is seen on a video screen with what is seen on the sea chart. Porathe concluded it is better on manned vessels to guide the operators by visualizing a 3D map oriented to match the user's view of the surrounding world [20]. Figure 1 shows that we have built our GUI corresponding with this research, as the user will see if the real-world corresponds to the 3Dworld, and thereby the position, easily and instantly. Moreover, if the system's position is not entirely accurate, the user can enhance the position accuracy by providing new bearing updates to the positioning system.

Our main contribution is to provide a GUI design for ship teleoperation providing good situation awareness, which meets the limitation of ships with a low throughput connection. We have shown that the users experienced the GUI to be simple to use while having a good overview of the situation. When the positioning system estimated an inaccurate position, the users could react upon this instantly. Furthermore, we have shown that our TAN application can be supported remotely by an operator taking bearings to landmarks.

This paper is organized as follows: Section II describes the Implementation and Method of the project, including the design of the applications in Subsection II-A, the Field Trial in Subsection II-B, and the User Study in Subsection II-C. The results are given in Section III, followed by Discussion and Conclusion in Section IV and V.

\section{IMPLEMENTATION AND METHOD}

This section describes how the software for the teleoperation tool and the positioning tool, called 
TAN application, have been designed and implemented, followed by a description of the field trial and user study.

\subsection{Design}

In our study, we focus on a GUI for teleoperation of a small ASV via a connection with limited throughput, which inhibits the transfer of video or high-resolution images. The ASV is expected to be semi-autonomous to handle a SAR mission but is still assumed to need some human supervision to take measures if something unexpected happens.

We have developed the GUI to suit a small ASV with a computer capacity and sensor suite comparable to an autonomous car. The postulated sensors and capabilities are:

- Global Positioning System (GPS), (only used for ground truth in the study, as we want to test the system using the redundant navigation system).

- A redundant navigation system, which can estimate the global position. In our case, this has been accomplished by fusion of compass and speed log data with data from a particle filter (PF) comparing available maps with bottom depth and magnetic intensity [9].

- Camera with $360^{\circ}$ coverage.

- Satellite communication system with a small antenna, providing a bandwidth of 256kbps.

- Application for cropping and compressing images, so that the ship can transmit panoramic images with a frequency of $0.1 \mathrm{~Hz}$, as well as an image with enhanced quality in the operator's direction, with a frequency of $1 \mathrm{~Hz}$.

\subsubsection{System as a whole - Architectural Overview}

An autonomous ship contains multiple subsystems, all interacting with each other to create a smart system that can perceive its environment and act upon it. In this project, we focus on two subsystems, the teleoperation tool to remote control an ASV, and the TAN application, which is used as a redundant positioning source to complement the GPS. The two subsystems are important on their own, but we evaluate how they can interact and benefit from each other in this project. How does the position estimation influence the user experience of the teleoperation tool, and how can the teleoperation tool strengthen the TAN application's performance?

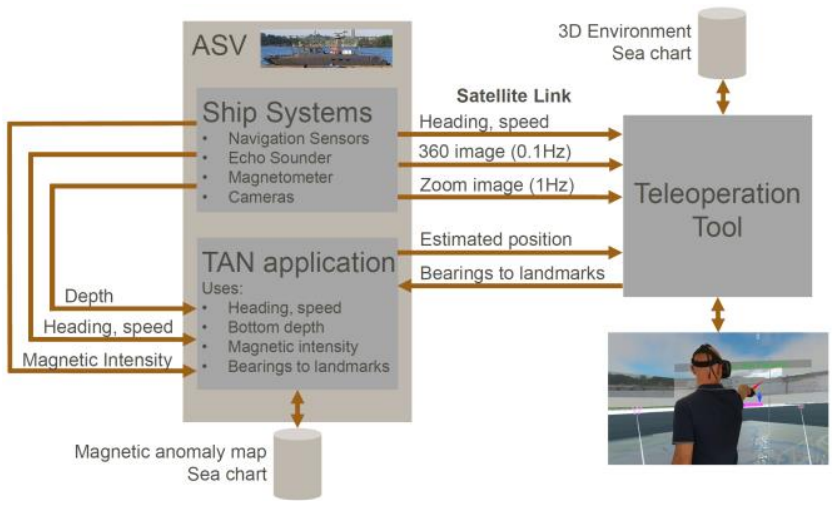

Figure 2 shows the information flows between the subsystems. The TAN application will run on a computer onboard the ASV, making it possible to receive all ship data in real-time. The teleoperation tool receives heading and speed together with cropped and compressed images. The TAN application sends the estimated position to the teleoperation tool, which transfers bearings to landmarks in return to the TAN application. The TAN application uses these bearings together with the heading, speed, depth, magnetic field, and the pre-loaded sea chart and magnetic anomaly map. All the interfaces between the TAN application and the teleoperation tool will be transmitted through a satellite link.

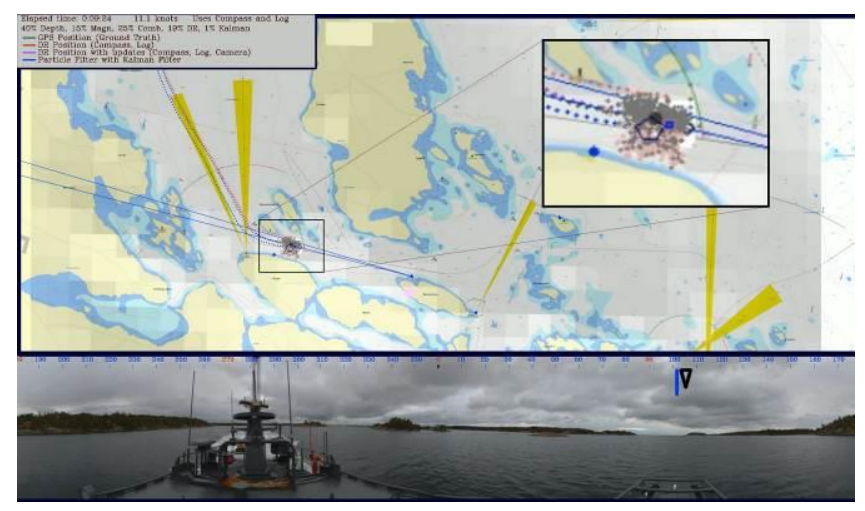

Figure 3. The GUI of the TAN application. The upper right corner shows an enlargement of the current operation area. The ship is going in the east direction. The particles are visualized as grey and pink dots, where the pink dots have just been discarded due to being outside of the bearing's cone towards the lighthouse in bearing $107^{\circ}$. The blue dot in the middle of the enlarged image indicate ground truth, which comes from the GPS. The large grey dot indicate the mean of the particles, and the pentagon the estimated position from the Kalman filter.

\subsubsection{Terrain-Aided Navigation}

The project described in this paper builds upon our previous work with a TAN application, which estimates the position by using a particle filter $(\mathrm{PF})$ to compare known maps to depth and magnetic intensity measurements [9]. We concluded that the position estimation gains accuracy when using multiple information sources instead of only using either depth or magnetic intensity separately. Figure 3 shows a screenshot of the GUI. For a better understanding, a video recording from the user study can be found on YouTube (https://youtu.be/zu40PEsk5cQ). The upper part of the figure shows the sea chart with an enlargement of the image showing the particles estimating the ship's position. The lower part shows the $360^{\circ}$ image. In the bearing $107^{\circ}$ in the $360^{\circ}$ image, a bearing to a landmark has just been detected. This is shown in the sea chart as a long blue cone originating from the landmark in the middle of the figure and stretching towards the ship. To satisfy the bearing measurement, the particles outside the cone are discarded, indicated as pink dots. In the user study, this GUI has been used for evaluating the TAN application's performance. The participants have not used it.

Figure 2. An architectural overview of the system. 
Tests showed that the positioning gained in performance from using the bearings to landmarks. These bearings were measured offline manually from the high-resolution images, which will not be possible in an unmanned vessel. A more realistic scenario is to use either image recognition software to detect landmarks, or that a human marks the landmarks from a remote location using low-quality images. In this project, we use the latter approach, where the user detects the landmarks in VR from a remote location.

\subsubsection{Graphical User Interface for teleoperation}

We have implemented the GUI for the teleoperation tool in Unity 3D [22], which game developers usually use for creating 2D and 3D games. We have used a $3 \mathrm{D}$ replica of the real world as a foundation for implementing the GUI. This 3D environment has been developed from maps and sea charts by the shipyard Saab Kockums AB [23].

The operator teleoperating the ship is virtually placed on board the virtual ship, positioned in the 3D environment according to where the TAN application is estimating the position. The tool receives speed and heading from the remote ship, which are used to move the vessel between each GUI frame. The $360^{\circ}$ image is updated only six times every minute to minimize the bandwidth usage. The zoom image, which is an image with better quality in the pointing direction, is updated every second. The GUI presents the images with some latency to simulate the slow satellite communication link.

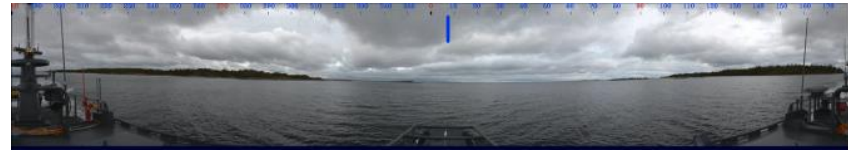

Figure 4. A frame of the high resolution (16384x8192) $360^{\circ}$ video, recorded during the field trial.

The GUI uses the panoramic video from the field trial both to create the zoom image and the $360^{\circ}$ image. The video quality is high, with $16384 \times 8192$ pixels of resolution. Figure 4 illustrates this with an example image. The problem is that the size of the images that build up the video is large and can not be transmitted in real-time over a satellite connection with low throughput. To meet the limitations, we have cropped and compressed the images. Figure 5 shows both the zoom image and the $360^{\circ}$ image in the teleoperation GUI, where the $360^{\circ}$ image surrounds the user and the zoom image is in the direction of the pointing device. The zoom image is presented in front of the $360^{\circ}$ image so that the better quality image covers the lower quality image. It slowly moves away from the user and vanishes behind the $360^{\circ}$ after a few seconds. If, e.g., holding the pointer steadily towards a specific object, one new image in that direction will appear every second.

To minimize the throughput, the $360^{\circ}$ image is sent with $0.1 \mathrm{~Hz}$ and the zoom image is sent with $1 \mathrm{~Hz}$. The compressed panoramic image has a size of around $30 \mathrm{kB}$, and the compressed zoom image with higher quality has a size of $3 \mathrm{kB}$. This results in a throughput of $6 \mathrm{kB} / \mathrm{s}$, or $48 \mathrm{kbps}$, which is a lot less than the $256 \mathrm{kbps}$ capacity of the communication link, leaving room for more user interface features.

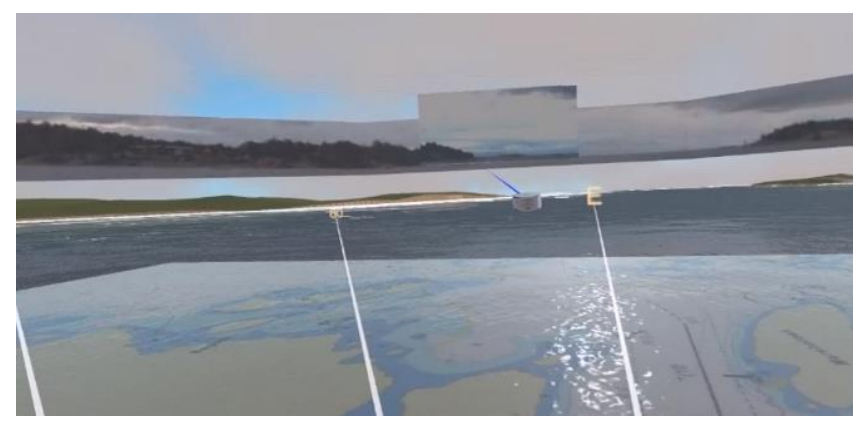

Figure 5. The GUI version called GUI ${ }^{\text {without }}$, without augmented landmarks. In the image the user is to take a bearing towards the shore.

\subsubsection{Two variants of the Graphical User Interface}

There are two variants of the GUI, GUI without and GUI ${ }^{\text {with }}$, each variant tested by half of the user study group. Figure 5 shows the variant without augmented landmarks called GUI $I^{\text {without }}$, where the user shall try to match objects between the upper $360^{\circ}$ image with objects in the lower 3D environment, without any augmented landmarks that guide the user. In the figure, the user is pointing towards the shore, which is also found below in the 3D environment. By first shooting the laser towards the specific point of the shore in the 3D environment and then towards the $360^{\circ}$ image, the application knows the ship is located in the opposite direction from the shore's specific position. This information is sent to the TAN application, which adjust the PF's position estimation accordingly.

This benefit of the GUI without's design is that the user is free to use all landmarks that can be found. The disadvantage is that it is quite difficult to point the laser pointer to the exact location in the vertical direction. If pointing a little bit over the intended direction, the user is pointing towards a position further away, which will result in the wrong position estimation. Another disadvantage with GUI without is that the users must be more creative and find the landmarks themselves.

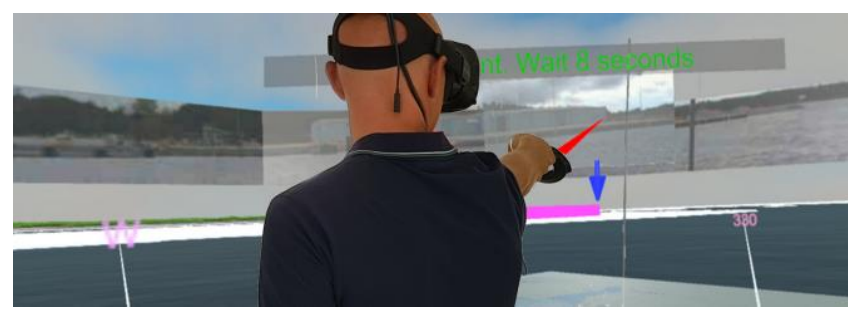

Figure 6. The GUI version called GUIwith, with augmented landmarks. (The user is overlaid over the image.)

Figure 6 presents the other variant of the GUI with augmented landmarks, called GUI with. The difference is that GUI with shows proposed landmarks as large pink markers, often with arrows, that turn blue when the user point towards them. The user in the image first shoots the laser towards the arrow (that points towards the right part of the house), and then directly at the right part of the house in the $360^{\circ}$ image. The tool then knows the landmark's exact position, in 
contrast to GUIwithout, where the user could slightly miss the target. A disadvantage is that the user is limited to the usage of only the proposed landmarks. Another screenshot from GUI with is shown in Figure 1.

\subsection{Field Trial}

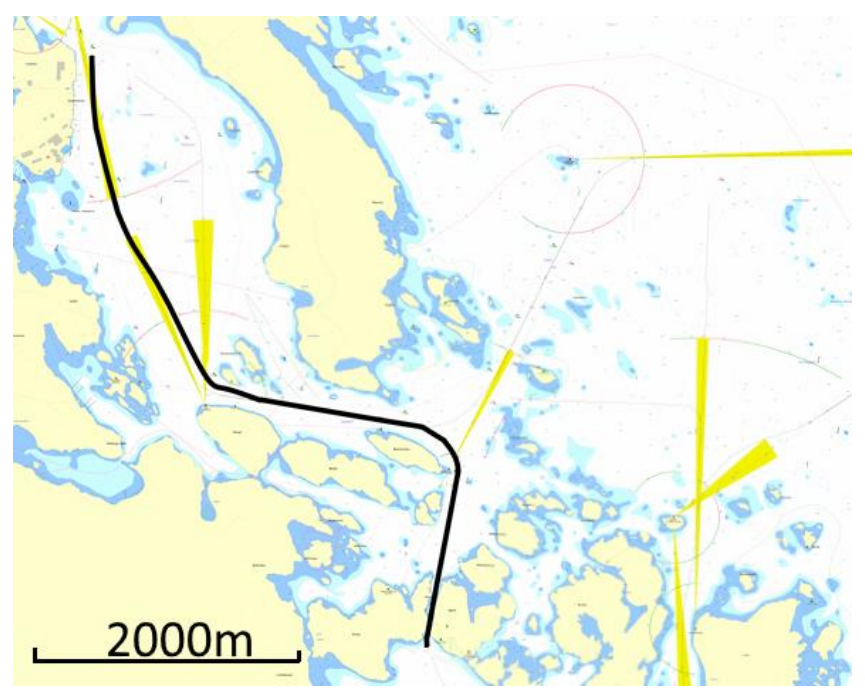

Figure 7. The route of the field trial overlaid on the sea chart. The boat went in the south-east direction.

We conducted a field trial in Västervik archipelago in Sweden to validate our approach, see Figure 7. We have used this field trial to collect data, which we have used for simulations and teleoperation tests. By using simulations for the user study instead of running the user study on the realworld ship, we have had the exact same scenario for all participants, making the results comparable. The boat used in the field trial is of type CB90, see Figure 8. It has been complemented with additional sensors to support the Swedish Universities via the WASP program [24] with a research platform for developing autonomous ships. We believe that the sensors onboard, see Table I, correspond to a sensor suite of a typical future affordable autonomous ship. We collected data from the digital compass onboard, but the ship was not equipped with a speed log. Instead, a virtual speed log was created using data from the GPS and adding an error of 0.2 knots (i.e., $\mathrm{NM} / \mathrm{h}$ ) to simulate a worst-case scenario. In the last step, to study the robustness of the algorithm's ability to navigate, we added a drift of a constant 0.5 knots to mimic the drift from the wind and current that can not be detected by the compass and speed log. The drift speed can, in general, be estimated with quite a good accuracy, and these 0.5 knots should be seen as the error between the estimated drift and the correct drift. If we can show that the TAN application can manage an inaccuracy of the drift speed of 0.5 knots, we believe the system is very robust. As the particle filter is estimating the position and not velocity nor the drift speed, the algorithm's results are not helped by a constant drift. The boat traveled a $9.2 \mathrm{NM}(17 \mathrm{~km})$ long route in $54 \mathrm{~min}$, but to make the user-study more manageable, we only used the first 20 minutes for the study.

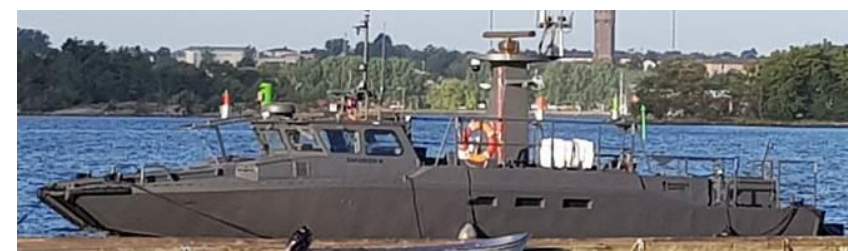

Figure 8 . The boat of type CB90 used in the field trial.

Table 1. Sensors used during field trial

\begin{tabular}{ll}
\hline Sensor & Description \\
\hline Compass $_{\text {Speed Log* }}$ & $\begin{array}{l}\left.\text { Heading (Accuracy } 0.5^{\circ}\right)-1 \mathrm{~Hz} \\
\text { Speed Through Water (STW) (Accuracy } \\
1 \%+0.1 \mathrm{knots})-1 \mathrm{~Hz}\end{array}$ \\
Echosounder & $\begin{array}{l}\text { Depth from surface to sea bed (Accuracy } \\
0.1 \mathrm{~m})-1 \mathrm{~Hz}\end{array}$ \\
Magnetometer & $\begin{array}{l}\text { Magnetic Intensity measured as a vector - } \\
\text { 100Hz }\end{array}$ \\
$360^{\circ}$ camera & $\begin{array}{l}\text { Provides visual image of the horizon } \\
\text { around most of the ship. Can alternatively } \\
\text { be multiple cameras. Images from 6 } \\
\text { cameras were compiled into an image }\end{array}$ \\
& with a resolution of $16384 \times 8192-15 \mathrm{~Hz}$ \\
\hline *The digital compass and speed log could be exchanged to \\
an INS.
\end{tabular}

More information regarding the data collected from the field trial can be found in our earlier work [9], where we used the data to evaluate the performance of the TAN application when using various fusion methods.

We conducted the whole study in an office by using the collected data. No ship was teleoperated for real, but the teleoperation interfaces were restricted to accommodate the low throughput connection.

\subsection{User Study}

We evaluated our implementations with a user study of 16 participants, recruited mainly from Lund University and the shipyard Saab Kockums AB, in 20minute long trial sessions with the task and scenarios described below. We recorded what the users saw in VR, as well as the TAN application GUI and its performance data. We informed the participants of the possibility of withdrawing at any time, and they agreed upon the use of screen recordings and other data for research purposes. Lund University ethics council did not require reviews of this kind of study since no personal data was studied. Four of the user study videos can be seen on YouTube (https://youtu.be/HwnIPuX-Azg, https://youtu.be/zu4 0PEsk5cQ, https://youtu.be/PCkAQhyAC6Q, and http s://youtu.be/HTm2GEZsxh0). The videos show both the teleoperation GUI and the TAN application GUI for two of the users. One of the users used GUI without (without augmented landmarks), and the other used GUI with (with augmented landmarks).

After an introduction phase based on written instructions and a quick oral summary, the user used the GUI in VR to remotely supervise the ship. The main task was to point towards the same objects in both the virtual 3D environment and the $360^{\circ}$ panoramic image, resulting in a bearing to a landmark. The teleoperation tool sent these bearings 
to the TAN application, which updated and increased the position's accuracy. Half of the subjects were randomly assigned using the GUI with, and the other half were assigned the GUI without where they had to find the landmarks by themselves. Our main questions to evaluate in the study were:

- Do the bearings from the operators increase the position accuracy for the TAN application, despite the low quality of the $360^{\circ}$ images?

- Do users experience they gain trust in the system's ability to navigate?

- Did users gain or lose performance when augmenting specific landmarks that the operators were to take bearing measurements from, instead of letting the operators freely pick landmarks that they thought would be good?

We compared the mean position error for each participant's recorded data from the user study (objective results).

After the experiments, the participants evaluated the GUI subjectively by answering the following four questions on a scale of 1-10 (1 was best on the first, and ten was best on question two to four):

1 It was difficult to handle the tool.

2 I experienced that I had a good overview of the situation.

3 If the tool further evolves, I believe that a real ship can be teleoperated using this technique.

4 If I practice 100 hours, my ability to use the tool would enhance further.

\section{RESULTS}

We have summarized the collected data from the user experiments in the objective and subjective results below, followed by some observations. We have interpreted the results in Section IV.

\subsection{Objective Results}

It is possible to estimate a ship's position by dead reckoning (DR) the position by using the compass and speed log. The problem with this method is that the error increases with time, as each measurement is based on the previous measurement, leading to a position error being accumulated over time. The TAN application uses a PF to compare the bottom depth and magnetic intensity with available maps to estimate the position more accurately. With this approach, the position error is not supposed to increase with time but, instead, holds its position relatively close to the correct position. We have Kalman filtered (KF) the mean of the PF's particle cloud. The KF provides a smoother and more accurate position estimation compared to only using the mean of the PF's cloud as a position estimation. By using bearings to landmarks, it is possible to reset the DR or PF's position estimation in the bearing direction by moving the particles or the DR estimation to the closest point in the bearing's direction, see Figure 3 or any of the YouTube videos for an example.

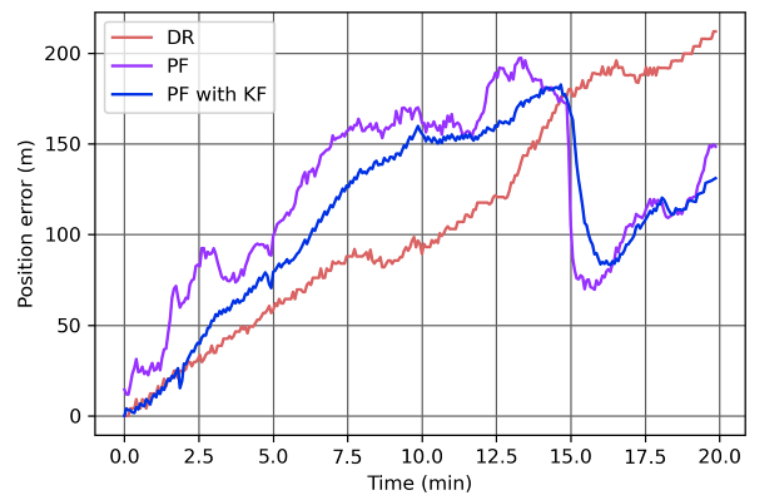

Figure 9. Position accuracy when not receiving any bearings to landmarks at all. In this particular example, the $\mathrm{PF}$ performance is worse than DR the first 15 minutes. This can happen as the PF use the DR as basis for its calculation, spreads the particles randomly, and then corrects the particle positions by comparing the maps with sensor measurements. In this particular situation, the maps matches the measurements quite well for the first $15 \mathrm{~min}$, despite the position being $150 \mathrm{~m}$ off. After $15 \mathrm{~min}$, the bottom depth do not match any more, which results in an adjustment of the particle cloud's mean position.

In the following, we present graphs and statistical analysis of the position error performance. The mean values are summarized in Table II. As we based the user study on a recording from a field trial, the DR without any bearing updates is the same for all user tests.

Table 2. Mean position error without bearings or with bearings from GUI without or GUI with

\begin{tabular}{llll}
\hline Algorithm & $\begin{array}{l}\text { Without } \\
\text { bearings }\end{array}$ & $\begin{array}{l}\text { Bearings from } \\
\text { GUI without }\end{array}$ & $\begin{array}{l}\text { Bearings from } \\
\text { GUI with }\end{array}$ \\
\hline DR & $111.6 \mathrm{~m}$ & $71.8 \mathrm{~m}$ & $57.6 \mathrm{~m}$ \\
PF & $123.5 \mathrm{~m}$ & $51.4 \mathrm{~m}$ & $42.8 \mathrm{~m}$ \\
PF with KF & $109.1 \mathrm{~m}$ & $36.5 \mathrm{~m}$ & $34.9 \mathrm{~m}$ \\
\hline
\end{tabular}

Figure 9 shows how the position error varies when not using any bearings to landmarks at all. As seen in the plot, the DR position error (red line) increased with time to around $200 \mathrm{~m}$ after $20 \mathrm{~min}$. The purple line, showing the position error when using PF, peaks after $13 \mathrm{~min}$ on about $200 \mathrm{~m}$. The accuracy is enhanced by Kalman filtering the PF (blue line), which peaks on about $175 \mathrm{~m}$. The mean error for DR was $111.6 \mathrm{~m}$, and the mean error for PF with KF was $109.1 \mathrm{~m}$. We can now use the value from PF with KF and see how the performance increases when the application receives bearings to landmarks.

Figure 10 shows a graph constructed from the eight GUI without user trials. The graph presents the averaged position error over the 20 -minute test. The DR position error increases with time (red line), but as the user takes bearings, the DR error is reset in the direction from the bearing (yellow line). As shown by the blue line, the KF corrected PF's mean error peaks at about $65 \mathrm{~m}$ after $7 \mathrm{~min}$. The mean error of the bearing-updated DR was $71.8 \mathrm{~m}$ (yellow line), and the bearing-updated PF with KF was $36.5 \mathrm{~m}$ (blue line). 


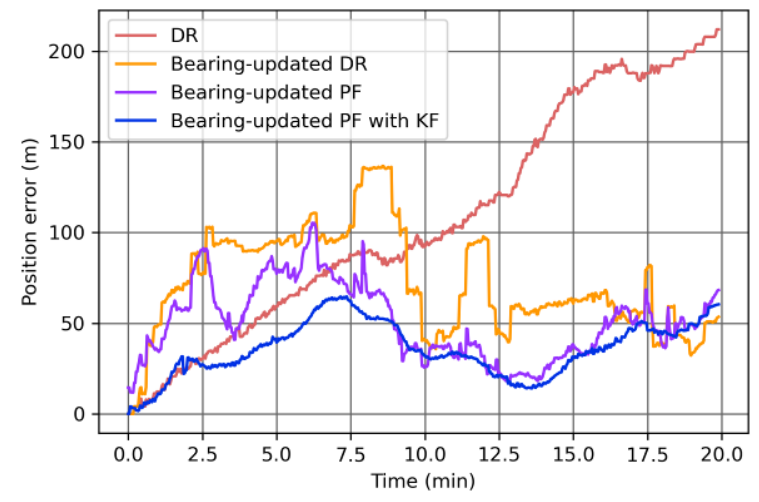

Figure 10. Position accuracy averaged from eight user trials using GUI without, compared to DR when not using any bearings at all (red line).

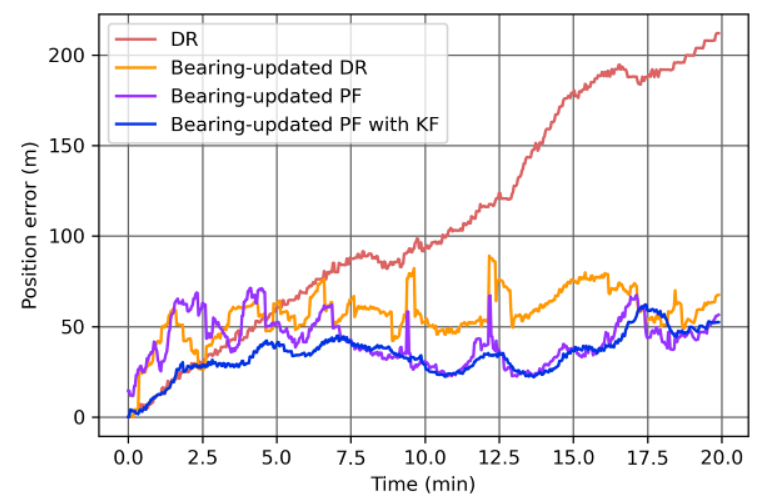

Figure 11. Position accuracy averaged from eight user trials using GUI with.

Figure 11 shows a graph constructed from the eight GUI with user trials. As shown by the blue line, the bearing-updated PF with KF mean error is relatively stable around $35-40 \mathrm{~m}$. The mean error of the bearingupdated DR was $57.6 \mathrm{~m}$ (yellow line). The bearingupdated PF with KF was $34.9 \mathrm{~m}$ (blue line), and peaked after about $17 \mathrm{~min}$ on about $60 \mathrm{~m}$.

We found that the tests with both the GUI without and GUI with significantly improved position accuracy by updating the TAN application with the bearings

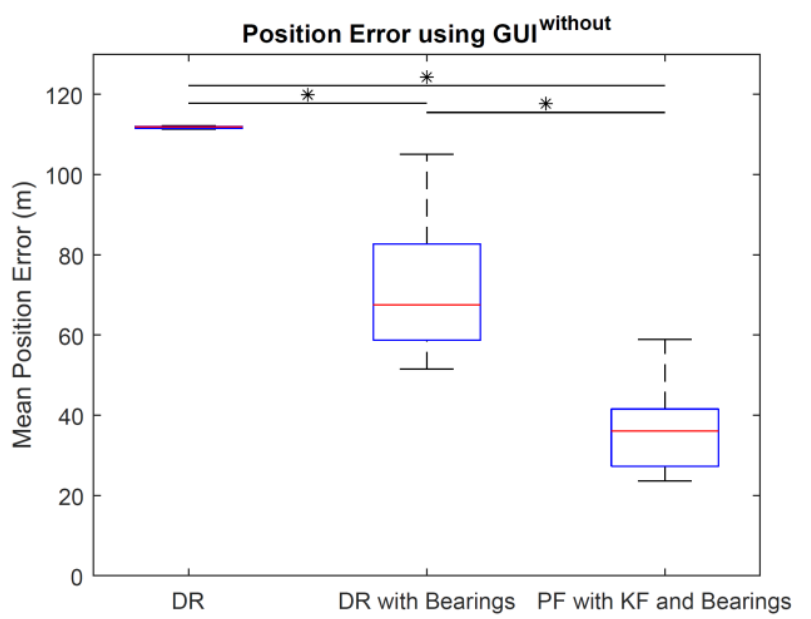

towards the landmarks. It was significantly better to use the bearing-updated PF with KF than just the bearing-updated DR, but also significantly better with bearing-updated DR compared to only DR (without bearings).

Figure 12 summarizes the objective results, along with the p-values showing the significant difference, computed in a series of one-tailed t-tests. The mean values are presented in Table II. Even though it was quite a small user study, power tests (alfa $=0.05$, power $>0.80$ ) have shown that there were enough participants to support the significant results. However, there was no significant difference between the two user groups.

\subsection{Subjective Results}

Table 3. Subjective results summary

\begin{tabular}{lll}
\hline Question & GUI without & GUI $^{\text {with }}$ \\
\hline Difficult to Manage* & 3.3 & 2.9 \\
User Overview & 6.3 & 6.9 \\
Applicability for real-world usage & 8.5 & 8.3 \\
Gain from Training & 8.4 & 8.9 \\
\hline
\end{tabular}

"Lower score is better.

For the user evaluation's subjective results, the users using GUI with gave better scores to all four questions except the question regarding the applicability for real-world usage. In general, though, the results were quite similar, and there have not been any significant differences. The scores, with a scale of 1-10 (1 was best on the first, and ten was best on question two to four), are presented in Table III. The users answered that the tool was not challenging to use (3.3 and 2.9) (lower score is better). They also had a good overview of the situation (6.3 and 6.9). Even more importantly, they believed a real-world ship would be possible to teleoperate in this way if the tool was further developed (8.5 and 8.3). They thought they would be even better handling the teleoperation tool after 100h of usage (8.4 and 8.9). Figure 13 summarizes the subjective results.

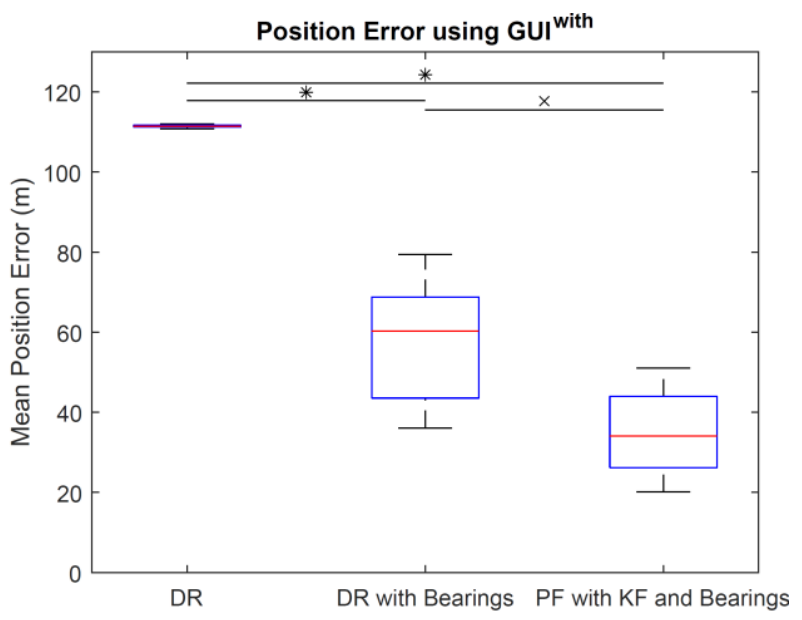

Figure 12. The objective results summarizing the position accuracy when using the different methods. $(\mathrm{x})$ and $\left(^{*}\right)$ denote comparisons with $p_{i} 0.01$ and $p_{;} 0.001$ respectively. Because we based the user study on a recording from a field trial, DR (to the left) is the same for all tests. 

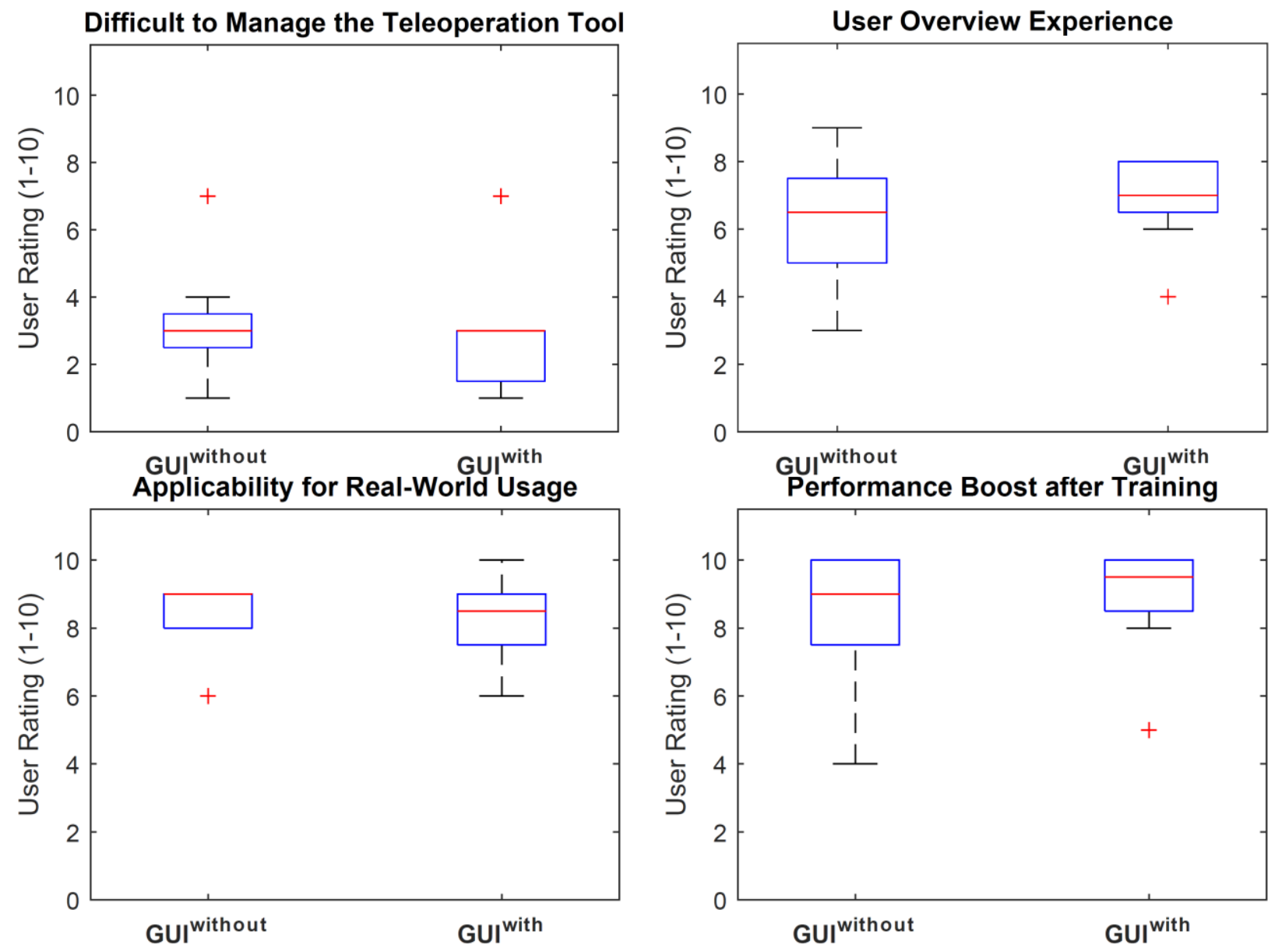

Figure 13. Subjective results from the questionnaire. Users answered the following questions: (a) It was difficult to handle the tool. (Lower score is better.) (b) I experienced that I had a good overview of the situation. (User Overview Experience) (c) If the tool further evolves, I believe that a real ship can be teleoperated using this technique. (Applicability for Real-World Usage) (d) If I practice 100 hours, my ability to use the tool would enhance further. (Performance Boost after Training)

We also asked the users to elaborate on good and bad aspects about the tool and how it felt using it.

Starting with the point that users wanted to be enhanced, most users (11 of 16) wanted either better resolution, higher frame-rate, or better lightning of the $360^{\circ}$ image. Two persons lacked support for glasses, as the head-mounted display HTCVive is of the older type where glasses do not fit. One participant suggested adding support for taking a bearing during the turning of the ship, which we have not implemented yet. When the shore was close to the vessel, the $3 \mathrm{D}$ environment could cover the $360^{\circ}$ image, which one person pointed out to aggravate the usability. There were also suggestions to enhance the $3 \mathrm{D}$ visualizations of buildings. Two persons found it a fun experience, while one person got bored after a while.

We asked the users about their VR experience, where the value 10 meant they were very experienced, and 1 meant they had no experience, resulting in the mean value of only 3.1. Still, 12 of the participants wrote that it was easy to use, easy to understand, or intuitive. Some had a problem understanding the tool during the first minutes but then said that they quickly learned. Three persons said they had either a good overview of the situation or that it was easy to orient, and another said that it was easy to find out if the estimated position did not match the $360^{\circ}$ image. One person reflected that he did not get any motion sickness, which surprised him, as he usually gets motion sickness from VR.

\subsection{Observations}

By observing the user's behavior during the study, it became clear that the users of GUIwithout, in general, had more difficulties at the beginning of the study, as they needed to orient themselves and learn quickly what type of landmarks would be appropriate to pick. Some of them got stressed, especially initially, and some participants took very few bearings during the first 5 minutes. After this initial period, these users seemed to have learned the teleoperation tool and were very creative in finding new landmarks to improve the position accuracy.

The users of GUI with, in general, figured out quite early what to do. Most of them acted quite skilled and took many bearings towards these fixed landmarks. We believe this task was not as challenging as with GUIwithout, and some of the participants looked a bit bored after a while. Being bored indicate low cognitive load, leaving room for conducting other tasks simultaneously. For not having even higher positioning performance, we consider a large reason to be the difficulty of hitting the GUI without's landmark accurately in the vertical direction with the laser pointer. We discovered from the saved video files from the user study that the laser pointer has many times pointed at an object behind the intended object, 
resulting in lower performance. To get better accuracy from using the GUI with, it should have been beneficial with more augmented landmarks, as the users could have handled many more.

\section{DISCUSSION}

What we mainly wanted to see in this study was if the users could get good situational awareness and feel that they were positioned in the correct location by being able to easily compare the $360^{\circ}$ image with the $3 \mathrm{D}$ environment. The questionnaire results, together with what the users wrote they experienced, have confirmed both these hypotheses. This is important, as it is difficult for a remote user to know if the position is accurate in a typical teleoperation system. Furthermore, the users have been given a good overview of the situation by using the $360^{\circ}$ image. Relatively small buoys more than $500 \mathrm{~m}$ away have been easily discovered.

We also wanted to confirm that the TAN application could gain in performance using bearings that the user took remotely. We did not know this beforehand, as we knew that the images would have relatively low quality, given the communication link with poor throughput.

We also wanted to know if there were any considerable differences between the two GUI types, which the user study did not imply. Still, the different GUI versions have given us some insights, as there were different advantages and disadvantages of the versions.

To enhance the implementation further, we have learned that:

- The $360^{\circ}$ image already has a low quality; hence it is helpful to increase the visibility as much as possible by, e.g., increasing the brightness.

- By using an augmented landmark to point towards, it is possible to get a more accurate position of the landmark, which is beneficial for the positioning system.

- Users are creative and can keep track of many objects. Do not limit the number of available augmented landmarks too much.

- The $360^{\circ}$ image should not be covered by the 3D environment, even when the ship is very close to shore.

Some users wanted a higher resolution and framerate for the $360^{\circ}$ image, which can be achieved when there is a better communication connection available with higher throughput. In this study, the throughput was very limited, though, as we wanted to see if it worked in the worst-case scenario.

The study provides knowledge about multiple aspects about how to create a teleoperation tool for an autonomous vessel, but the user-study has not intended to evaluate a complete system design. We believe more research is needed for this. We still do not know if VR is a good solution for multiple hours of operations, and we believe a final design for expert users should be designed in a different way, optimized for the intended usage and scenario.

\section{CONCLUSION AND FUTURE WORK}

We have developed and tested a GUI for the teleoperation of an affordable Autonomous Surface Vehicle using a low throughput connection. Our findings show that users have had a good overview despite the low-quality images. The users have experienced the position as correctly estimated by easily matching the $3 \mathrm{D}$ environment with the $360^{\circ}$ image. When it did not match, they quickly have reacted and tried to solve the problem by updating the positioning system with new bearings. We can conclude that the positioning system has increased its accuracy by using these bearings, despite the lowquality link connection.

Together with the results from our previous work [10], [11], which focused on features to provide good situational awareness and safety, while maintaining a low cognitive load, we now believe we have all functions needed to combine all building blocks into a more comprehensive GUI with more complexity, tailored for trained expert users. Building upon this, we aim to conduct a new user study with expert users teleoperating the ship while having safety drivers on board to meet current regulations.

\section{ACKNOWLEDGMENT}

This work was partially supported by the Wallenberg AI, Autonomous Systems and Software Program (WASP) [24] funded by Knut and Alice Wallenberg Foundation. Saab Kockums AB [23] provided the ship used for the field trial.

\section{REFERENCES}

[1] Kongsberg. (2018) Rolls-royce and finferries demonstrate world's first fully autonomous ferry. Last accessed 10 October 2020. [Online]. Available: https://www.rolls-royce.com/media/press-

releases/2018/03- 12-2018-rr-and-finferries-demonstrateworlds-first-fully-autonomousferry. aspx

[2] Kongsberg. (2018) Technology for the ferries of the future. Last accessed 10 October 2020. [Online]. Available: https://www.kongsberg.com/maritime/aboutus/news-andmedia/ news-archive/2018/technology-forthe-ferries-of-the-future/

[3] (2018) Remote-controlled and autonomous ships in the maritime industry. Last accessed 9 September 2020. [Online].

Available: https://www.dnvgl.com/maritime/publications/remotecontrolledautonomous- ships-paper-download.html

[4] O. Levander, "Autonomous ships on the high seas," IEEE Spectrum, vol. 54, no. 2, pp. 26-31, 2017.

[5] C. Lundberg, H. I. Christensen, and A. Hedstrom, "The use of robots in harsh and unstructured field applications," in ROMAN 2005. IEEE International Workshop on Robot and Human Interactive Communication, 2005. IEEE, 2005, pp. 143-150.

[6] Y. Zheng, M. J. Brudnak, P. Jayakumar, J. L. Stein, and T. Ersal, "Evaluation of a predictor-based framework in high-speed teleoperated military UGVs," IEEE Transactions on Human-Machine Systems, 2020.

[7] R. Luz, J. Corujeira, L. Grisoni, F. Giraud, J. L. Silva, and R. Ventura, "On the use of haptic tablets for UGV teleoperation in unstructured environments: System design and evaluation," IEEE Access, vol. 7, pp. 95 43195 442, 2019. 
[8] R. Murphy, "Disaster Robotics. Intelligent robotics and autonomous agents series," The MIT Press, 2014.

[9] M. Lager, E. A. Topp, and J. Malec, "Robust terrainaided navigation through sensor fusion," in 2020 IEEE 23rd International Conference on Information Fusion (FUSION). IEEE, 2020, pp. 1-8.

[10] M. Lager, E. A. Topp, and J. Malec, "Remote operation of unmanned surface vessel through virtual reality-a low cognitive load approach," in Proceedings of the 1st International Workshop on Virtual, Augmented, and Mixed Reality for HRI (VAM-HRI), 2018.

[11] M. Lager and E. A. Topp, "Remote supervision of an autonomous surface vehicle using virtual reality," IFAC-PapersOnLine, vol. 52, no. 8, pp. 387-392, 2019.

[12] S. Neumeier, N. Gay, C. Dannheim, and C. Facchi, "On the way to autonomous vehicles teleoperated driving," in AmE 2018-Automotive meets Electronics; 9th GMMSymposium. VDE, 2018, pp. 1-6.

[13] S. Neumeier, P. Wintersberger, A.-K. Frison, A. Becher, C. Facchi, and A. Riener, "Teleoperation: The holy grail to solve problems of automated driving? Sure, but latency matters," in Proceedings of the 11th International Conference on Automotive User Interfaces and Interactive Vehicular Applications, 2019, pp. 186197.

[14] S. Lu, M. Y. Zhang, T. Ersal, and X. J. Yang, “Workload management in teleoperation of unmanned ground vehicles: Effects of a delay compensation aid on human operators' workload and teleoperation performance," International Journal of Human-Computer Interaction, vol. 35, no. 19, pp. 1820-1830, 2019.

[15] A. Hosseini, F. Richthammer, and M. Lienkamp, "Predictive haptic feedback for safe lateral control of teleoperated road vehicles in urban areas," in 2016 IEEE 83rd Vehicular Technology Conference (VTC Spring). IEEE, 2016, pp. 1-7.

[16] F. Chucholowski, M. Sauer, and M. Lienkamp, "Evaluation of display methods for teleoperation of road vehicles," Journal of Unmanned System Technology, vol. 3, no. 3, pp. 80-85, 2016.

[17] T. Williams, N. Tran, J. Rands, and N. T. Dantam, "Augmented, mixed, and virtual reality enabling of robot deixis," in the International Conference on Virtual, Augmented and Mixed Reality, 2018.

[18] A. Hosseini and M. Lienkamp, "Enhancing telepresence during the teleoperation of road vehicles using hmd-based mixed reality," in Intelligent Vehicles Symposium (IV), 2016 IEEE. IEEE, 2016, pp. 1366-1373.

[19] X. Shen, Z. J. Chong, S. Pendleton, G. M. J. Fu, B. Qin, E. Frazzoli, and M. H. Ang, "Teleoperation of on-road vehicles via immersive telepresence using off-the-shelf components," in Intelligent Autonomous Systems 13. Springer, 2016, pp. 1419-1433.

[20] T. Porathe, "User-centered map design," in Usability Professionals' Association Conference, 2007.

[21] S. Witt, "Technologies used for navigation," Novel Interaction Techniques for Oceangoings, University of Passau, 2017.

[22] (2018) Unity 3d. Last accessed 29 September 2020. [Online]. Available: https://unity.com/

[23] (2020) Saab Kockums AB. Last accessed 29 September $2020 . \quad$ [Online]. Available: https://www.saab.com/products/naval

[24] (2020) WASP. Last accessed 29 January 2020. [Online]. Available: http://wasp-sweden.org/ 